\title{
A new approach for extracting and scoring aspect using SentiWordNet
}

\author{
Tuan Anh Tran, Jarunee Duangsuwan, Wiphada Wettayaprasit \\ Artificial Intelligence Research Lab, Department of Computer Science, Division of Computational Science, \\ Faculty of Science, Prince of Songkla University, Songkhla, Thailand
}

\begin{tabular}{l}
\hline Article Info \\
\hline Article history: \\
Received Feb 10, 2021 \\
Revised Mar 17, 2021 \\
Accepted Mar 20, 2021 \\
\hline
\end{tabular}

Keywords:

Aspect extraction

Aspect scoring

Score level

SentiWordNet

\begin{abstract}
Aspect-based online information on social media plays a vital role in influencing people's opinions when consumers concern with their decisions to make a purchase, or companies intend to pursue opinions on their product or services. Determining aspect-based opinions from the online information is necessary for business intelligence to support users in reaching their objectives. In this study, we propose the new aspect extraction and scoring system which has three procedures. The first procedure is normalizing and tagging part-of-speech for sentences of datasets. The second procedure is extracting aspects with pattern rules. The third procedure is assigning scores for aspects with SentiWordNet. In the experiments, benchmark datasets of customer reviews are used for evaluation. The performance evaluation of our proposed system shows that our proposed system has high accuracy when compared to other systems.
\end{abstract}

This is an open access article under the CC BY-SA license.

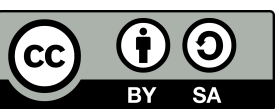

Corresponding Author:

Wiphada Wettayaprasit

Department of Computer Science, Division of Computational Science

Faculty of Science, Prince of Songkla University, Songkhla, Thailand

Email: wiphada.w@psu.ac.th

\section{INTRODUCTION}

Nowadays, the digital era affects humans' behaviors in choosing reference resources to decide their decisions. The online information usually composes of opinions or feelings expressed by the Internet users about services, healthcare, products, politics, etc. Determining and understanding the Internet users' opinions (e.g., happy or unhappy) using sentiment analysis is the vital key-role to apply to marketing, and making decisions or recommendations [1-3].

In textual online information, the users usually mention about opinions or feelings. These attributes are called aspects, and the phase to extract the useful aspects from the online information is called aspect extraction [4-8]. In the previous works, most of these studies extracted aspects from customers' reviews and did not show how much satisfied or dissatisfied the Internet users mentioned in reviews for the aspects. To determine and understand how much satisfied or dissatisfied the Internet users mention for aspects is useful to make decisions. In this study, we propose aspect extraction and scoring system (AESS) to extract and score aspects which become the knowledgebase. Datasets from independent domains (e.g., services, products, etc.) are the input of the AESS. The pre-processing phase is normalizing and tagging part-of-speech (POS). The AESS uses pattern rules to extract aspects from datasets. SentiWordNet is used to assign score levels for aspects. The output is the scored aspect knowledgebase which shows satisfied levels of the users as well.

The rest of the paper is organized as the following: The related works are presented in section 2 . The 
architecture of the proposed AESS system is discussed in section 3. The experimental results and evaluation are explained in section 4. Finally, the conclusion is given in section 5.

\section{RELATED WORK}

To extract aspect, Wei et al. [9] proposed semantic-based product feature extraction (SPE) method which used the association rule mining algorithm to extract aspects. Qiu et al. [10] presented a double-propagation (DP) algorithm which used dependency relations among constituencies in a sentence to extract aspects. Liu et al. [4] extended more dependency relations $\left(\mathrm{DP}^{+}\right)$to extract aspects. Rana and Cheah [11] proposed a two-fold rules-based model (TF-RBM) which used sequential pattern rules to extract aspects. Mataoui et al. [12] introduced a method for the Arabic language by using syntactic rules in order to extract aspects. Rana and Cheah [13] proposed a sequential pattern rules-based approach (SPR) to automatically produce sequential pattern rules to extract aspects. Poria et al. [14] suggested rules and dependency trees to extract aspects (explicit and implicit). Meanwhile, Alqaryouti et al. [15] used rules to extract aspects (explicit and implicit) from government reviews.

For aspect scoring, Kherwa et al. [16] assigned a score for an aspect by calculating an average score of opinion words from SentiWordNet where these opinion words and that aspect co-occurred. Asghar et al. [17] chose the highest score in three scores (positive, negative, objective) of an opinion word which were respective average scores of all synsets of that opinion word from SentiWordNet. Xu et al. [18] used frequency and a dictionary to calculate scores. Jmal and Faiz [19] calculated a score by using the popularity of a frequency for one aspect on Twitter and scores (negative, positive, neutral) from SentiWordNet of words (verb/adjective) related to the aspect. The frequency of the aspect was estimated in the dataset. Meanwhile, Maheswari and Dhenakaran [20] used a dictionary for opinion words and Fuzzy rules.

\section{PROPOSED METHODOLOGY}

To automatically extract and score aspects from datasets, the AESS is proposed and illustrated in Figure 1. The AESS system has three procedures: 1) pre-processing, 2) aspect extraction using pattern rules and Word2Vec, and 3) aspect scoring using SentiWordNet. The input of the system is datasets such as product reviews. The output of the system is the scored aspect knowledgebase which can be represented in graphics.

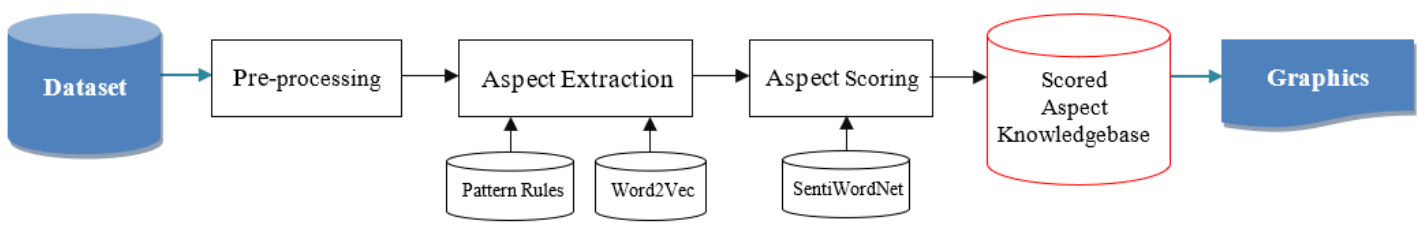

Figure 1. An architecture of AESS

\subsection{Pre-processing}

This procedure aims to normalize and tag POS for sentences of datasets. The details are 1) eliminating special characters in the text of social media, e.g., HyperText markup language (HTML) tags, a pair of quotations, 2) correcting misspelt words, and 3) tagging POS for text.

\section{2. $\quad$ Aspect extraction}

This procedure is used to extract aspects with opinion words and intensifier words from datasets using pattern rules. There are two main steps: 1) aspect candidates extraction, and 2) aspect pruning.

Let $a$ be an aspect, ow be an opinion word in the (opinion lexicons) OL, and $i w$ be an intensifier word. Let neg be a negation status which shows a negation word existing in a sentence with an opinion word where neg $\in\{$ True, False $\}$.

Definition 1: Sentence based on aspect-opinion-intensifier (SAOI) is a set which members have a quadruple $<a, o w, i w, n e g\rangle$ in the sentence as shown in (1)

$$
\mathrm{SAOI}=\left\langle a_{i}, o w_{i}, i w_{i}, \text { neg }_{i}\right\rangle
$$

where $i$ is an index of an extracted aspect, $1 \leq i \leq \mathrm{n}, \mathrm{n}$ is the number of extracted aspects. 
- Step 1. aspect candidates extraction. This step will extract aspect candidates from datasets by using the pattern rules and the OL dictionary (Bing Liu's opinion lexicon [21] and MPQA's opinion lexicon [22]). After extracting, the aspects, opinion words, and intensifier words are saved in SAOI. The pattern rules are determined by using the relationship between aspect and opinion words. The relationships based on a syntactic structure are determined from the dependency tree [23]. Some examples of the pattern rules are in Table 1. There are opinion word(s) in italic, aspect(s) in bold, co-reference word(s) in italic bold, optional words in brackets, and a subscript showing positions for a constituent in a sentence (e.g., "a", "b", etc.).

- Step 2. aspect pruning. This step eliminates the irrelevant aspects by using the cosine similarity and Word2Vec (Word2Vec is provided by SpaCy [24]).

Table 1. Some examples of pattern rules for aspect extraction

\begin{tabular}{|c|c|c|c|}
\hline Pattern No. & Syntax-based Pattern Rule & Pattern No. & Syntax-based Pattern Rule \\
\hline S1 & $A P+\mathbf{C N}$ & S6 & $\mathbf{C N}+\mathrm{RCl}+\mathrm{V} 2 \mathrm{~A}+A P($ Note: $\mathrm{RCl}$ is any pattern $)$ \\
\hline $\mathrm{S} 2$ & $\mathbf{C N}+R C l$ & S7 & $\begin{array}{l}\mathbf{C N}_{\mathbf{a}}+\mathrm{V}+(\text { Prep })+\mathrm{CN}_{\mathrm{b}}+\mathrm{V} 2 \mathrm{~A}+A P \\
\text { Note: Prep is "by"; } \mathrm{V} \text { is } \mathrm{V}+\mathrm{ed} / \mathrm{V}+\mathrm{ing}\end{array}$ \\
\hline S3 & $\mathrm{V} 2 \mathrm{~A}+(\mathrm{Adv})+A 2+\mathbf{N P}$ & S8 & $\begin{array}{l}\text { Pron }_{1}+\mathrm{V} / \mathrm{V} 2 \mathrm{~A}+\mathbf{C N}+(\mathrm{Adv})+\mathrm{Conj}+\boldsymbol{P r o n}_{2}+\mathrm{V} 2 \mathrm{~A}+A P \\
\text { Note: } \text { Pron }_{2} \text { is a co-reference of } \mathrm{CN}\end{array}$ \\
\hline S4 & $\mathbf{C N}+\mathrm{V} 2 \mathrm{~A}+A P$ & S9 & $(A d v)+V 2+\mathbf{N P}$ \\
\hline S5 & $\mathbf{C N}_{a}+\mathrm{V} 2 \mathrm{~A}+C N_{b}$ & S10 & $\mathbf{C N}+\mathrm{V} 2 \mathrm{~A}+V($ Note: $\mathrm{V}$ is $\mathrm{V}+\mathrm{ed} / \mathrm{V}+\mathrm{ing})$ \\
\hline
\end{tabular}

\subsection{Aspect scoring}

A goal of this procedure is to score aspects by using SentiWordNet (SentiWordNet which is a lexical resource is automatically annotated "positivity" and "negativity" scores for all of synsets [25]).

Definition 2: Opinion value of an opinion word $(O V)$ is an average of all synsets values for an opinion word (ow) which are retrieved from SentiWordNet as shown in (2)

$$
O V= \begin{cases}\left(\sum_{i=1}^{\mathrm{p}} P V_{i}\right) / \mathrm{p} & , \text { if } \text { ow } \in \mathrm{OLP} \\ \left(\sum_{i=1}^{\mathrm{p}} N V_{i}\right) / \mathrm{p} & , \text { if } \text { ow } \in \mathrm{OLN}\end{cases}
$$

where $\mathrm{p}$ is a number of entries (synsets) for ow in SentiWordNet, $P V_{i}$ is the $i^{t h}$ positive value, $N V_{i}$ is the $i^{t h}$ negative value, OLP is a set of Opinion Lexicons in Positive (e.g., "good", "great", etc.), and OLN is a set of Opinion Lexicons in Negative (e.g.,"bad", "hate", etc.) (OL = OLP $\cup$ OLN).

Definition 3: Sentence polarity $(\mathrm{SPol})$ is a value which is aggregated from a negation status neg and an opinion word (ow) in a sentence as shown in (3)

$$
\text { SPol }= \begin{cases}+1 & , \text { if polarity }{ }_{\text {ow }} \bigoplus n e g=\text { True } \\ -1 & , \text { if polarityow } \bigoplus \text { neg }=\text { False }\end{cases}
$$

where neg is a negation word exists in a sentence or not, and polarity $y_{o w}$ is a polarity of an opinion word (polarity $_{o w}$ is equal to True if an opinion word (ow) is positive. polarity pw $_{\text {is }}$ is equal to False if an opinion word (ow) is negative).

For example, from the sentence "A picture is not beautiful", an opinion word "beautiful" is positive. Polarity of "beautiful" is determined True. A negation word is "not". neg for "not" is True. With polarityow = True and neg $=$ True, polarityow $\bigoplus n e g$ and SPol equal to False and -1, respectively. Let $I V_{i w}$ be an Intensifier Value of an intensifier word $(i w) . I V_{i w}$ is pre-defined by users in Table 2 and has the value in $[-1$, $1]$.

Definition 4: SAOI score for an aspect $\left(\right.$ SScore $\left._{a}\right)$ is a value which is aggregated from values of an opinion word, an intensifier word, and negation expressed by users' opinions for aspect $a$ in one sentence as shown in (4).

$$
S_{S c o r e}=S P o l \times\left[\left(I V_{i w} \times O V\right)+O V\right]
$$

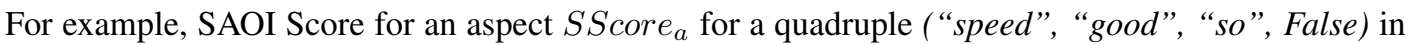
Table $3(i=1)$ from the sentence "The speed is so good" is calculated with Formula (4) as follows: "good" is positive opinion (i.e. polarityow $=$ True). $S P o l=+1$ because $n e g=$ False and polarityow $=$ True. 
Intensifier word "so" has intensifier value 0.45 (i.e. $\left.I V_{i w}=0.45\right) . O V$ for "good" is an average score which is retrieved from SentiWordNet and is equal to 0.70. Hence, $S$ Score $e_{a}=(+1) \times[(0.45 \times 0.70)+0.70]=1.02$. The example of $S$ Score $e_{a}$ for aspects are shown in Table 3.

Table 2. Intensifier values $\left(I V_{i w}\right)$ for intensifier words $(i w)$

\begin{tabular}{lclc}
\hline \multicolumn{1}{c}{ Intensifier word(s) } & $\begin{array}{c}\text { Intensifier Value } \\
(i w)\end{array}$ & \multicolumn{1}{c}{$\begin{array}{c}\text { Intensifier word(s) } \\
\left(I V_{i w}\right)\end{array}$} & $\begin{array}{c}\text { Intensifier Value } \\
\left(I V_{i w}\right)\end{array}$ \\
\hline awfully, critically & -1.00 & altogether, so & 0.45 \\
dangerously, dreadfully, hopelessly & -0.70 & primarily, very & 0.50 \\
bitterly, horribly, strikingly & -0.50 & highly & 0.55 \\
terribly, violently & -0.50 & largely, reasonably & 0.60 \\
suspiciously, slightly & -0.40 & greatly & 0.65 \\
somewhat & -0.25 & hugely, surprisingly, totally, utterly & 0.70 \\
mildly, quite & -0.20 & fully, mainly, deeply & 0.70 \\
faintly & 0.10 & especially, particularly, predominantly & 0.75 \\
really, purely & 0.15 & amazingly, exceedingly, extremely & 0.80 \\
remarkably, nearly, partly & 0.20 & incredibly, seriously, unbelievably & 0.80 \\
pretty, rather, roughly & 0.20 & wonderfully, exclusively & 0.80 \\
simply & 0.25 & entirely, almost, mostly & 0.90 \\
fairly, moderately & 0.30 & absolutely, completely, perfectly & 1.00 \\
\hline
\end{tabular}

Table 3. Examples of $S$ Score $e_{a}$ and score level for aspects

\begin{tabular}{|c|c|c|c|c|c|c|c|c|c|c|}
\hline \multirow[b]{2}{*}{$i$} & \multicolumn{4}{|c|}{ SAOI } & \multirow[t]{2}{*}{ SPol } & \multirow[t]{2}{*}{$I V_{i w}$} & \multirow[t]{2}{*}{$O V$} & \multirow[t]{2}{*}{ SScore $_{a}$} & \multicolumn{2}{|c|}{ score level } \\
\hline & $a_{i}$ & $o w_{i}$ & $i w_{i}$ & neg $_{i}$ & & & & & number & name \\
\hline 1 & speed & good & so & False & +1 & 0.45 & 0.70 & 1.02 & +2 & very satisfied \\
\hline 2 & battery & good & “" & True & -1 & 0 & 0.70 & -0.70 & -2 & very dissatisfied \\
\hline 3 & battery & loved & "'” & False & +1 & 0 & 0.71 & 0.71 & +2 & very satisfied \\
\hline 4 & speed & bad & ""” & True & +1 & 0 & 0.66 & 0.66 & +1 & satisfied \\
\hline 5 & battery & cool & quite & False & +1 & -0.20 & 0.29 & 0.23 & +1 & satisfied \\
\hline 6 & battery & good & "” & False & +1 & 0 & 0.70 & 0.70 & +2 & very satisfied \\
\hline 7 & speed & bad & “"” & False & -1 & 0 & 0.66 & 0.66 & +1 & satisfied \\
\hline
\end{tabular}

Definition 5: Score level is a pair of two data (number, name) in which "number" is an integer number in $[-3,+3]$, and "name" is ("the most dissatisfied", "very dissatisfied", "dissatisfied", "so so", "satisfied", "very satisfied", "the most satisfied"). Relations between number and name are $\{(-3$, "the most dissatisfied"), (-2, "very dissatisfied"), (-1, “dissatisfied"), (0, "so so"), (+1, "satisfied"), (+2, "very satisfied"), (+3, "the most satisfied")\}.

Score level for an aspect $a\left(S L_{a}\right)$ is determined by using $S S$ core $e_{a}$ as shown in (5)

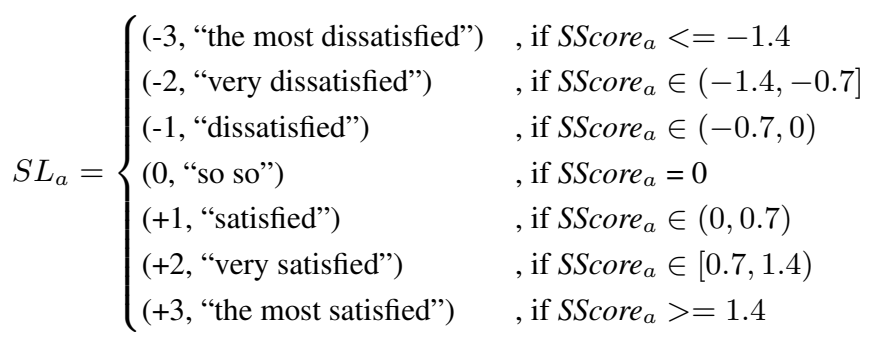

note that minimum and maximum scores of $S S$ core $e_{a}$ are -2 and +2 , respectively.

For example, $S$ Score f $_{a}$ for "speed" in the previous example ( $i=1$ in Table 3$)$ equals to 1.02. Score level for "speed" is (+2, "very satisfied"). Score levels for all of aspects are shown in the last two columns of Table 3.

Definition 6: Scored aspect knowledgebase (Sakb) is a set which members have an octuple $<a, l_{-3}, l_{-2}, l_{-1}, l_{0}, l_{+1}, l_{+2}, l_{+3}>$ as shown in (6)

$$
\left.\mathrm{Sakb}=<a_{k}, l_{-3}, l_{-2}, l_{-1}, l_{0}, l_{+1}, l_{+2}, l_{+3}\right\rangle
$$

where $k$ is an index of an aspect (none redundant), $1 \leq k \leq \mathrm{m}, \mathrm{m}$ is the number of none redundant aspects, $1_{\text {name }}$ is a frequency of score level for aspect $\mathrm{a}_{k}$. 
Algorithm 1 explains aspect extraction and scoring from a dataset. Line 1 is used to extract aspect and other information by using pattern rules and save into SAOI. Line 2 is used to eliminate irrelevant aspects by using the cosine similarity and Word2Vec. Line 3 is used to initialize Scored Aspect Knowledgebase (Sakb). In lines 4-10, the algorithm scores aspects in SAOI. If an aspect $a_{i}$ is not in Sakb, then a new aspect $a_{i}$ is added to Sakb. $l_{\text {number }}$ values are equal to 0 for initialization. $S S$ core $a_{i}$ is calculated for aspect $a_{i}$. The score level for aspect $a_{i}\left(S L_{a_{i}}\right)$ is calculated by using $S S$ core $a_{i}$ in (5). A frequency of score level for aspect $a_{i}$ at $l_{\text {number }}$ value is increased by 1 . On line 11, the algorithm returns Sakb.

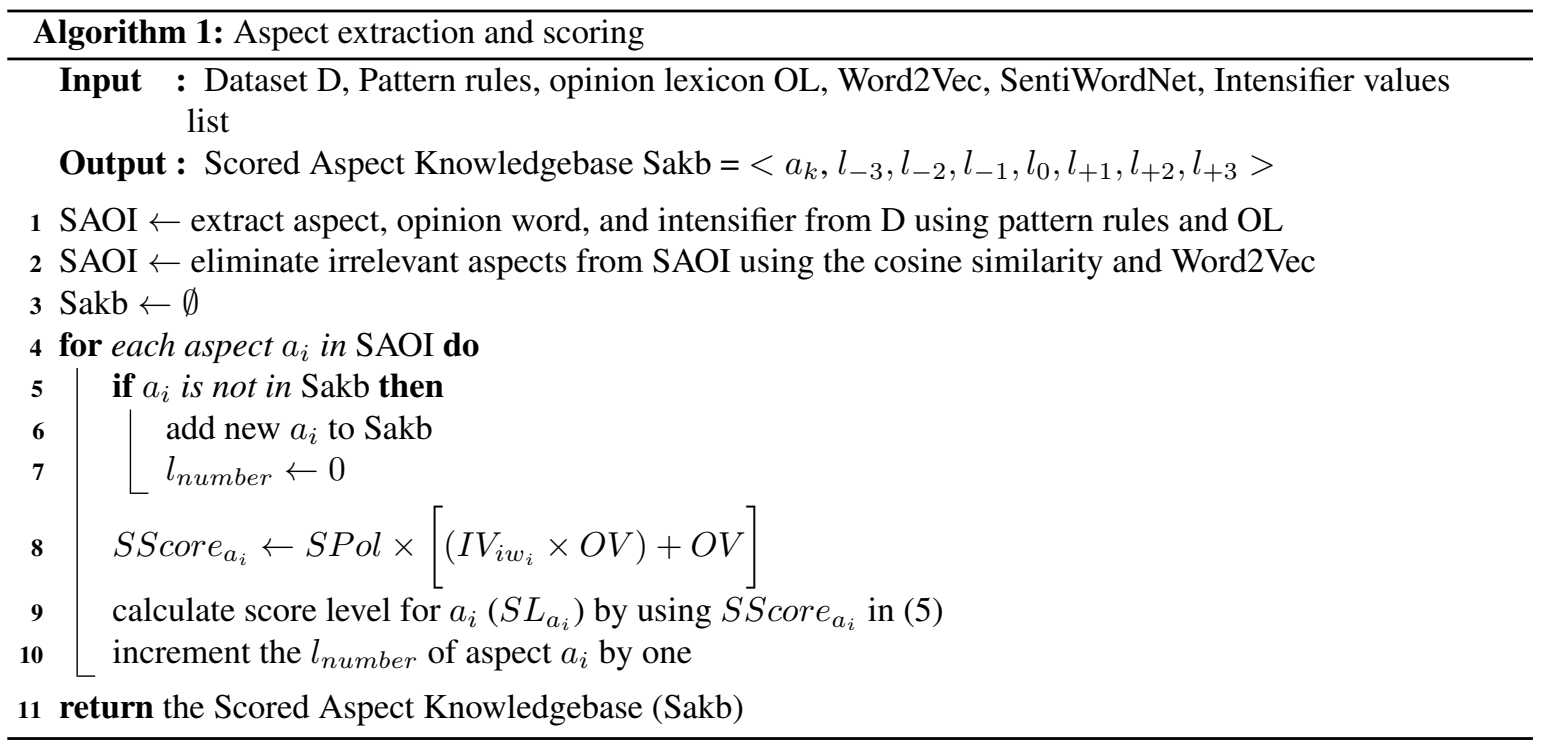

For example, the Aspect Extraction and Scoring algorithm has been applied to Table 3. The result has two tuples. Two aspects of the result are speed and battery. The Sakb values of $l_{\text {number }}$ for aspect speed are $<$ speed, $0,0,0,0,2,1,0>$. The Sakb values of $l_{\text {number }}$ for aspect battery are $<$ battery, $0,1,0,0,1,2,0>$.

\section{RESULT AND DISCUSSION}

In this study, we used two benchmark datasets to conduct our experiment. The first dataset [4] has three reviewed domains (computer, speaker, and router). The second dataset [21] has five reviewed domains (Canon camera, MP3 player, Nokia cellphone, Nikon camera,and DVD player). Each reviewed domain is described with the format reviewed_domain [total of sentences/ total of aspects] as the following: Computer [531/ 354], Speaker [689/440], Router [879/ 307], Canon camera [597/ 237], MP3 player [1,716/ 674], Nokia cellphone [546/302], Nikon camera [346/ 174], and DVD player [740/ 296].

In our experiment, the result is the scored aspect knowledgebase which is used to represent with graphical charts. In addition, we compare the proposed method with other approaches by using three measures (Precision, Recall, and F1-score) [13, 21]. The formulas are Precision $=T P /(T P+F P)$, Recall $=$ $T P /(T P+F N)$, and $F 1$-score $=(2 \times \mathrm{P} \times \mathrm{R}) /(\mathrm{P}+\mathrm{R})$, where $T P$ is $|E \cap A|, F P$ is $|E \backslash A|$, and $F N$ is $|A \backslash E|$. Note that $E$ is the set of extracted aspects, and $A$ is the set of annotated aspects in datasets. Figure 2 shows comparisons of the performance experimented with three measures (Precision, Recall, and F1-score). The comparisons are semantic-based product feature extraction (SPE) [9], double propagation (DP) [10], $\mathrm{DP}^{+}$[4], two-fold rule-based model (TF-RBM) [11], sequential pattern rule (SPR) [13], and the proposed AESS. From Figure 2, our proposed method AESS has the highest precision for all of the reviewed domains. In terms of F1-score, the proposed method shows the highest result for Computer, Speaker, Canon camera, and Mp3 player with the values 0.80, 0.74, 0.93, and 0.83, respectively. Furthermore, from AESS system Figure 3 shows some examples of graphical charts for Computer reviewed. Figure 3 a shows all aspects score with so so $80 \%$, satisfied $12 \%$, dissatisfied $7 \%$, and the most dissatisfied $1 \%$. Figure $3 \mathrm{~b}$ shows "screen quality" aspect score with satisfied $75 \%$ and dissatisfied $25 \%$ 


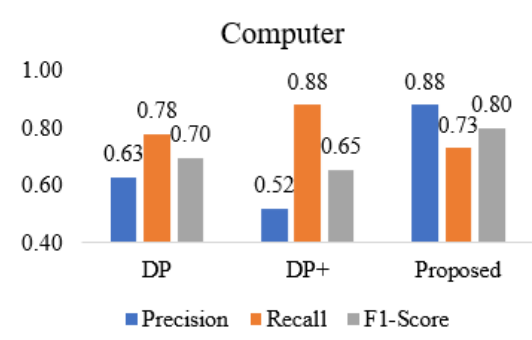

(a)

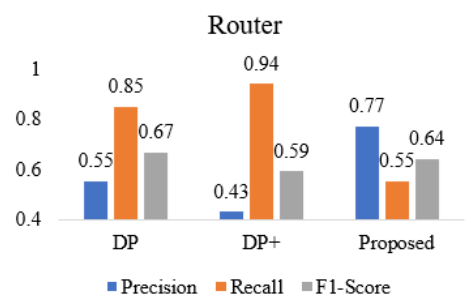

(c)

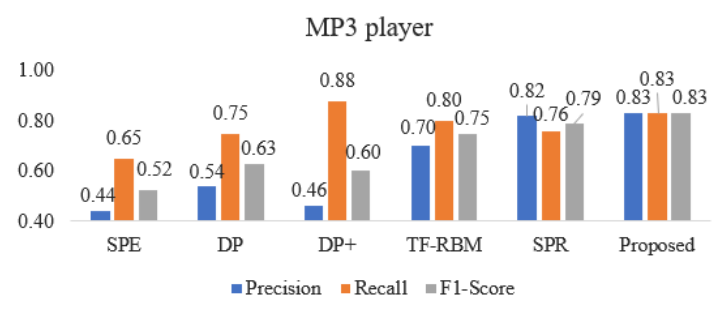

(e)

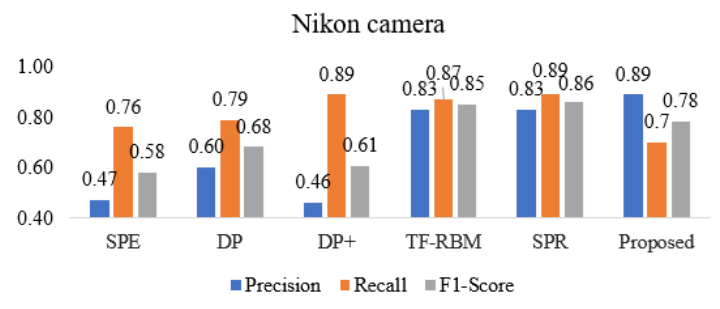

(g)

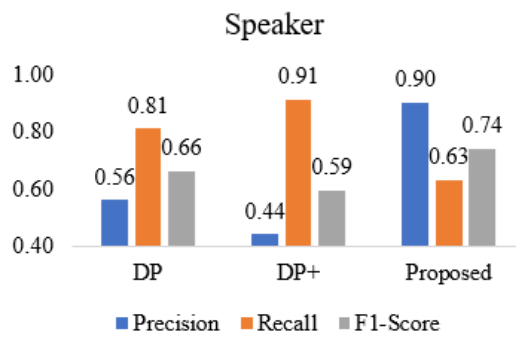

(b)

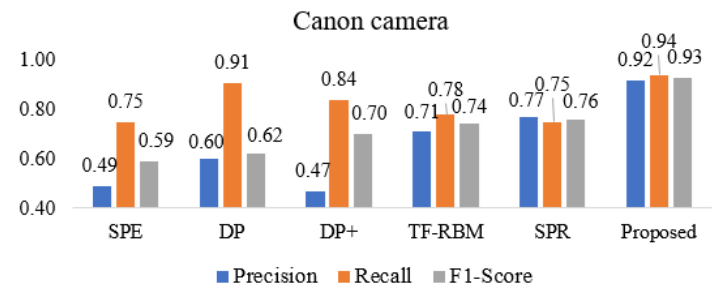

(d)

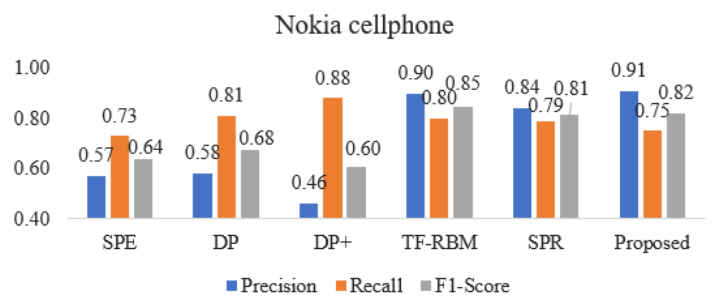

(f)

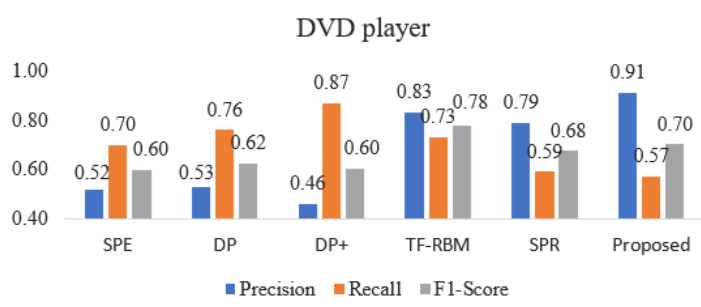

(h)

Figure 2. The comparison of approaches for reviewed domains: (a) computer, (b) speaker, (c) router,

(d) canon camera, (e) mp3 player, (f) nokia cellphone, (g) nikon camera, and (h) DVD player

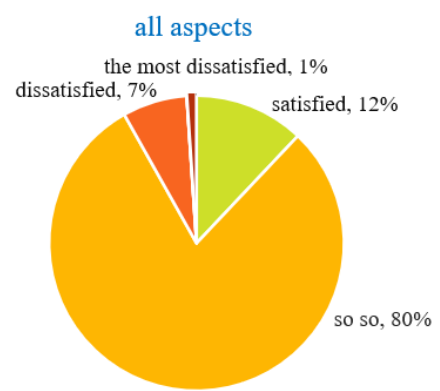

(a)

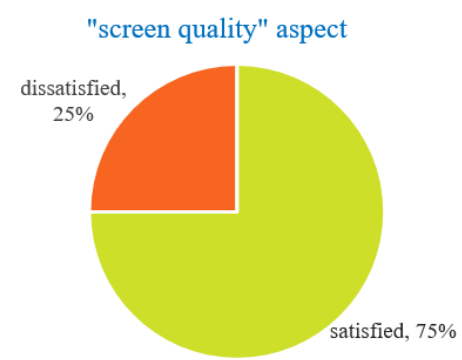

(b)

Figure 3. Graphical charts representing for computer reviewed domain: (a) all aspects score and (b) "screen quality" aspect score 


\section{CONCLUSION}

Customer satisfaction or dissatisfaction feedback is really important for business intelligent systems. We proposed the new aspect extraction and scoring system (AESS) to represent the satisfaction or dissatisfaction of the consumers in graphical format. The input of the AESS is the textual online data. The output of the AESS is the score of the aspect knowledgebase. The aspect knowledgebase is extracted by using pattern rules and assigned score levels with SentiWordNet. From the benchmark datasets, the proposed AESS has a very high performance when compared to other approaches.

The proposed AESS could be applied to independent domains (e.g., services, products, etc.). Moreover, the proposed AESS does not need any annotated data. In future work, we have a plan to retrieve scores from different lexical resources.

\section{ACKNOWLEDGEMENT}

This work was supported by Thailand's Education Hub for the Southern Region of ASEAN Countries (TEH-AC) and PSU.GS. Financial Support for Thesis (Fiscal Year: 2019).

\section{REFERENCES}

[1] A. A. Jihad and A. S. Abdalkafor, "A framework for sentiment analysis in Arabic text," Indonesian Journal of Electrical Engineering and Computer Science, vol. 16, no. 3, pp. 1482-1489, 2019.

[2] M. A. Ayu, S. S. Wijaya, and T. Mantoro, "An automatic lexicon generation for Indonesian news sentiment analysis: a case on governor elections in Indonesia," Indonesian Journal of Electrical Engineering and Computer Science, vol. 16, no. 3, pp. 1555-1561, 2019.

[3] B. Gliwa, A. Zygmunt, and M. Dabrowski, "Building sentiment lexicons based on recommending services for the Polish language," Computer Science, vol. 17, no. 2, pp. 163-185, 2016.

[4] Q. Liu, Z. Gao, B. Liu, and Y. Zhang, "Automated rule selection for opinion target extraction," Knowledge-Based Systems, vol. 104, no. 15, pp. 74-88, 2016.

[5] L. Bing, Sentiment Analysis: Mining Opinions, Sentiments, and Emotions. New York, NY 10013-2473, USA: Cambridge University Press, 2015.

[6] M. Pontiki, D. Galanis, H. Papageorgiou, S. Manandhar, and I. Androutsopoulos, "SemEval-2015 task 12: Aspect based sentiment analysis," in Proceedings of the 9th International Workshop on Semantic Evaluation. Denver, Colorado: Association for Computational Linguistics, Jun. 2015, pp. 486-495.

[7] Q. Liu, Z. Gao, B. Liu, and Y. Zhang, "Automated rule selection for aspect extraction in opinion mining," in Proceedings of the 24th International Conference on Artificial Intelligence, 2015, pp. 1291--1297.

[8] M. Pontiki, D. Galanis, J. Pavlopoulos, H. Papageorgiou, I. Androutsopoulos, and S. Manandhar, "SemEval-2014 task 4: Aspect based sentiment analysis," in Proceedings of the 8th International Workshop on Semantic Evaluation. Dublin, Ireland: Association for Computational Linguistics, Aug. 2014, pp. 27-35.

[9] C.-P. Wei, Y.-M. Chen, C.-S. Yang, and C. C. Yang, "Understanding what concerns consumers: a semantic approach to product feature extraction from consumer reviews," Information Systems and e-Business Management, vol. 8, no. 2, pp. 149-167, 2010.

[10] G. Qiu, B. Liu, J. Bu, and C. Chen, "Opinion word expansion and target extraction through double propagation," Computational Linguistics, vol. 37, no. 1, pp. 9-27, 2011.

[11] T. A. Rana and Y.-N. Cheah, "A two-fold rule-based model for aspect extraction," Expert Systems with Applications, vol. 89, no. 15 , pp. 273-285, 2017.

[12] M. Mataoui, T. E. B. Hacine, I. Tellache, A. Bakhtouchi, and O. Zelmati, "A new syntax-based aspect detection approach for sentiment analysis in Arabic reviews," in Proceedings of the 2nd International Conference on Natural Language and Speech Processing (ICNLSP), 2018, pp. 1-6.

[13] T. A. Rana and Y.-N. Cheah, "Sequential patterns rule-based approach for opinion target extraction from customer reviews," Journal of Information Science, vol. 45, no. 5, pp. 643-655, 2019.

[14] S. Poria, E. Cambria, L.-W. Ku, C. Gui, and A. Gelbukh, "A rule-based approach to aspect extraction from product reviews," in Proceedings of the 2nd Workshop on Natural Language Processing for Social Media (SocialNLP), 2014, pp. 28-37.

[15] O. Alqaryouti, N. Siyam, A. A. Monem, and K. Shaalan, "Aspect-based sentiment analysis using smart government review data," Applied Computing and Informatics, 2020.

[16] P. Kherwa, A. Sachdeva, D. Mahajan, N. Pande, and P. K. Singh, "An approach towards comprehensive sentimental data analysis and opinion mining," in 2014 IEEE International Advance Computing Conference (IACC), 2014, pp. 606-612. 
[17] M. Z. Asghar, A. Khan, S. R. Zahra, S. Ahmad, and F. M. Kundi, "Aspect-based opinion mining framework using heuristic patterns," Cluster Computing, vol. 22, pp. 7181-7199, 2017.

[18] X. Xu, T. Meng, and X. Cheng, "Aspect-based extractive summarization of online reviews," pp. 968-975, 2011.

[19] J. Jmal and R. Faiz, "Customer review summarization approach using Twitter and SentiWordNet," in Proceedings of the 3rd International Conference on Web Intelligence, Mining and Semantics, USA, 2013.

[20] S. U. Maheswari and S. S. Dhenakaran, "Aspect based fuzzy logic sentiment analysis on social media big data," in 2020 International Conference on Communication and Signal Processing (ICCSP), 2020, pp. 0971-0975.

[21] M. Hu and B. Liu, "Mining and summarizing customer reviews," in Proceedings of the 10th International Conference on Knowledge Discovery and Data Mining (SIGKDD). USA: ACM, 2004, pp. 168-177.

[22] T. Wilson, J. Wiebe, and P. Hoffmann, "Recognizing contextual polarity in phrase-level sentiment analysis," pp. $347-354,2005$

[23] A. Ranta, Grammatical Framework: Programming with Multilingual Grammars. CSLI Publications, Center for the Study of Language and Information, 2011.

[24] Spacy, "Spacy guides," 2020. [Online]. Available: https://spacy.io/

[25] S. Baccianella, A. Esuli, and F. Sebastiani, "Sentiwordnet 3.0: An enhanced lexical resource for sentiment analysis and opinion mining." $\quad$ European Language Resources Association, 2010.

\section{BIOGRAPHIES OF AUTHORS}

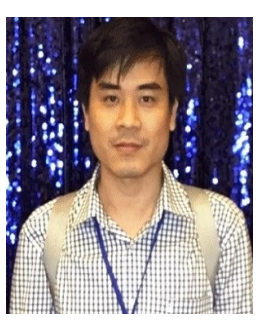

Tuan Anh Tran, is a PhD. candidate at Department of Computer Science, Division of Computational Science, Faculty of Science, Prince of Songkla University, Thailand. He got his BSc. in Information Technology from Hue University of Education, MSc. in Information System from Ho Chi Minh city University of Sciences, Vietnam. His research interest is Natural Language Processing.

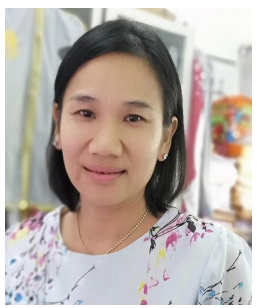

Jarunee Duangsuwan, is an assistant professor at Department of Computer Science, Division of Computational Science, Faculty of Science, Prince of Songkla University, Thailand. She got BSc., MSc., PhD. in Computer Science from Chiang Mai University, Prince of Songkla University, and University of Reading, UK, respectively. Her research interests are Natural Language Processing and Machine Learning.

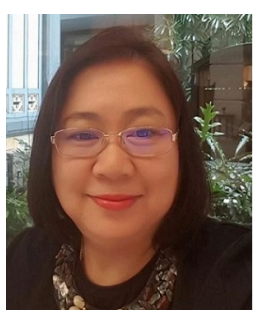

Wiphada Wettayaprasit, is an assistant professor at Department of Computer Science, Division of Computational Science, Faculty of Science, Prince of Songkla University, Thailand. She got BSc., MSc., PhD. in Computer Science from Prince of Songkla University, University of Missouri-Columbia, USA, and Chulalongkorn University, respectively. Her research interests are Artificial Intelligence, Neural Networks and Machine Learning. 